SYPHILIS

\title{
Relation between the HIV and the re-emerging syphilis epidemic among MSM in Germany: an analysis based on anonymous surveillance data
}

\author{
U Marcus, C Kollan, V Bremer, O Hamouda
}

Sex Transm Infect 2005;81:456-457. doi: 10.1136/sti.2005.014555

In 2003, for the first time since the introduction of highly active antiretroviral therapy (HAART), the number of newly diagnosed HIV infections in Germany increased considerably compared to the previous year. The increase was largely restricted to men who have sex with men (MSM) from larger cities. In this group the number of newly diagnosed HIV infections increased about $30 \%$ compared to 2002 . Since the late 1990s syphilis infections among MSM also increased in Germany, concentrated in larger cities.

$\mathrm{T}$ analyse the relation between the HIV and syphilis epidemics among men who have sex with men (MSM) in Germany and the possible impact of increasing syphilis rates on HIV epidemiology, we matched reports of newly diagnosed HIV and syphilis infections. Since 2001, both HIV and syphilis are notifiable anonymously by the diagnosing laboratory, using similar notification forms with common variables, including gender, month and year of birth, and the first three digits of the five digit postal code. Details of both notification systems are described elsewhere. ${ }^{2}$ Before the year 2001 only aggregated data were available for syphilis, and for HIV the month of birth was not recorded, therefore, matching was not possible for cases diagnosed before 2001.

An additional data source for our analysis is a sexually transmitted diseases (STD) sentinel surveillance system established in 2002. ${ }^{3}$ Approximately 10\% of syphilis notifications are covered by this system in parallel.

In respect of the time sequence of reported HIV and syphilis diagnoses we defined three distinct groups: (1) syphilis diagnosed in people with known HIV infection (HIV diagnosis precedes syphilis diagnosis by $>3$ months); (2) coincident diagnoses (syphilis and HIV infection are diagnosed within a time window of plus or minus 3 months), and (3) syphilis followed by an HIV diagnosis more than 3 months later.

There were 8497 cases of syphilis and 5712 cases of newly diagnosed HIV infection reported from January 2001 through June 2004. For the calculation of the HIV/syphilis overlap among MSM we used only male cases with reported transmission risk MSM or unknown $(\mathrm{MSM} / \mathrm{u})$, resulting in 6518 syphilis cases, including 1472 matching cases, and 3319 cases of HIV, including 1253 matching cases (difference in matching numbers caused by syphilis re-infections in HIV positives). To include matching cases, either from the HIV or the matching syphilis diagnosis, an MSM risk information or a concordant unknown risk was required.

The matching cases represent $23 \%$ of all syphilis diagnoses in $M S M / u$. The proportion of syphilis cases in all ever reported patients with known HIV infection cannot be determined, because HIV infections diagnosed before 2001 could not be matched (see above). The proportion of syphilis diagnoses in MSM/u patients with recent HIV diagnosis (since January 2001) reached $14 \%$ for syphilis cases diagnosed in 2003 (95\% CI: 13\% to 15\%) and 16\% for cases diagnosed in 2004 (95\% CI: 14\% to $18 \%$ ).

These proportions are in line with observations from our STD sentinel surveillance system, where the reported HIV prevalence in MSM patients with a new diagnosis of syphilis is in the range between $41 \%$ and $50 \% .^{3}$ The proportion of reported syphilis re-infections $(27 \%$ of 714 matches with respective classification) is significantly higher in patients with prevalent HIV compared to group 2 and 3 patients $(\mathrm{OR}=2.2,95 \% \mathrm{CI}: 1.6$ to 3.1$)$.

The proportion of syphilis cases with coincident HIV diagnosis increased from 5\% (2001) (95\% CI: $4 \%$ to $6 \%$ ) to $7 \%$ (2003) (95\% CI: $6 \%$ to $8 \%$ ) in MSM/u. Assuming an HIV prevalence of $50 \%$ among MSM patients with a new diagnosis of syphilis, the proportion of co-incident HIV infection in previously HIV negative or untested MSM with a new syphilis diagnosis may reach between $10 \%$ and $14 \%$ ( $12.7 \%$ in the sentinel system). In another approximately $20 \%$ of previously HIV negative or untested MSM with a new diagnosis of infectious syphilis in 2001, HIV was newly diagnosed in the following 2 years, representing an HIV incidence in this subgroup of MSM of about $10 \%$ per year.

The matching cases represent $38 \%$ of the newly diagnosed male HIV cases with MSM/u risk reported in the period January 2001-June 2004. The proportion of coincident diagnoses among all newly diagnosed HIV infections in MSM/u increased from $8 \%$ in 2001 to $14 \%$ (95\% CI: $13 \%$ to $15 \%)$ in the following 3 years. In an additional 9\% (95\% CI: $8 \%$ to $10 \%$ ) (2002) to $12 \%$ (2004) of newly diagnosed HIV infections in MSM/u, HIV diagnosis was preceded by the diagnosis of an infectious syphilis by more than 3 months, in the same or the previous year.

There are several limitations to our analysis: the matching procedure based on the limited data set is not perfect and HIV infections diagnosed before 2001 could not be included in the match. Different people of the same gender, year, and month of birth, living in the same postal code area could have been falsely matched and matching could have failed because of incorrect reports of any of the matching parameters (we experience variability especially of the last of the three postal code digits) and if people move to another postal code area. We would thus expect that we underestimate rather than overestimate the number of matching reports. In defining the denominator population we had to include male HIV and syphilis cases with unknown risk information, because in the matching cases the risk information for HIV and syphilis

Abbreviations: HAART, highly active antiretroviral therapy; MSM, men who have sex with men; MSM/u, MSM or unknown; STD, sexually transmitted diseases 


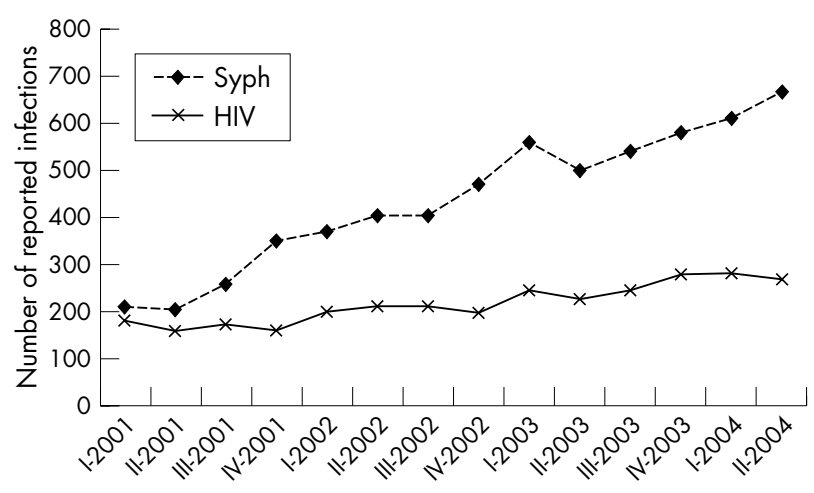

Figure 1 Reported cases of HIV and infectious syphilis among MSM in Germany by quarter of diagnosis.

reports was not always congruent. While the large majority of male HIV and syphilis cases with unknown risk in Germany are likely to be MSM, we may have included some male cases with other risk factors. This would, however, diminish rather than increase the proportion of matches.

Nevertheless, we believe that the following conclusions can be drawn.

There is a considerable overlap of syphilis and HIV epidemics among MSM. Sexual risk taking goes on after the diagnosis of HIV and/or syphilis in a sizeable proportion of MSM, therefore, appropriate risk reduction counselling for both HIV positive and HIV negative patients with syphilis is necessary. Special emphasis should be put on partner notification for patients co-diagnosed with HIV and syphilis, because they may be at high risk of having been exposed to both infectious pathogens or they may themselves be the source of the respective infections.

An increase of HIV transmission rates among MSM has been observed only several years after the re-emergence of syphilis among MSM in Germany. ${ }^{4}$ In terms of epidemiological synergy between HIV and syphilis, this suggests that the increase in newly diagnosed HIV infection may be rather a result of ongoing risk behaviour in an accumulating number of newly infected and still undiagnosed and/or untreated co-infected people than a result of HIV transmission from people with known and treated HIV infection. ${ }^{6}$ Owing to the changing perception of the usefulness of early treatment for HIV infection most of the men with recently diagnosed HIV infection will currently not receive antiretroviral therapy.
Finally, our results suggest that the relation between HIV and syphilis epidemics among MSM is complex. Increasing syphilis incidence among MSM may contribute to increasing transmission rates for HIV because of an "epidemiological synergy" between both infections. ${ }^{7}$ However, the relation between increasing incidence of syphilis and HIV is not necessarily always causal. MSM, who in earlier years may have "only" become infected with HIV, will now also become infected with syphilis because of the increasing prevalence of this pathogen ("epidemiological coincidence"). Causality may even be reversed, in the sense that HIV diagnosis may result in a change of the sexual network of newly HIVinfected people (that is, preference for HIV seroconcordant partners) with subsequent increased risk of syphilis acquisition and/or increased risk of syphilis re-infection as a result of a potentially reduced immunological resistance to re-infection caused by HIV induced immunological defects ("behavioural/immunological susceptibility"). ${ }^{89}$

\section{Authors' affiliations}

U Marcus, C Kollan, V Bremer, O Hamouda, Robert Koch-Institut, Berlin, Germany

Correspondence to: Ulrich Marcus, Robert Koch-Institut, Berlin, Germany; marcusu@rki.de

Accepted for publication 8 April 2005

\section{REFERENCES}

1 Marcus U, Bremer V, Hamouda O. Syphilis surveillance and trends of the syphilis epidemic in Germany since the mid-90s. Eurosurveillance Monthly. 2004;9, 11-14 (www.eurosurveillance.org/em/v09n12/0912-225.asp).

2 Hamouda O. HIV/AIDS surveillance in Germany. J AIDS 2003;32:S49-S54.

3 Bremer V, Marcus U, Hofmann A, et al. Building a sentinel surveillance system for sexually transmitted infections in Germany, 2003. Sex Transm Infect 2005:81:173-9.

4 CDC. Trends in primary and secondary syphilis and HIV infections in men who have sex with men - San Francisco and Los Angeles, California, 1998-2002. MMWR 2004;53:575-8.

5 Plettenberg A, Adam A, Weidner L, et al. Deutliche Zunahme der Syphilis bei HIV-Infizierten in Hamburg. 8. Berlin, German AIDS Congress, 2001 (www.ifi-infektiologie.de/informationen/aktuelles.html).

6 CDC. High-risk sexual behavior by HIV-positive men who have sex with men16 sites, United States, 2000-2002. MMWR 2004;53:891-4.

7 Buchacz K, Patel P, Taylor M, et al. Syphilis increases HIV viral load and decreases CD4 cell counts in HIV-infected patients with new syphilis infections. AIDS 2004; 18:2075-9.

8 Morgan CA, Lukehart SA, Van Voorhis WC. Protection against syphilis correlates with specificity of antibodies to the variable regions of Treponema pallidum repeat protein K. Infect Immun 2003;71:5605-12.

9 Grassly NC, Fraser C, Garnett GP. Host immunity and synchronized epidemics of syphilis across the United States. Nature 2005;433:417-21.

11 th European Forum on Quality Improvement in Health Care

26-28 April 2006, Prague, Czech Republic

For further information please go to: www.quality.bmipg.com

Book early to benefit from a discounted delegate rate 